\title{
Kallikrein 11 expressed in human breast cancer cells releases insulin-like growth factor through degradation of IGFBP-3
}

\author{
ATSUMI SANO ${ }^{1,2}$, TAKAFUMI SANGAI ${ }^{2}$, HIROYUKI MAEDA ${ }^{2}$, MICHIO NAKAMURA ${ }^{2}$, \\ TAKAHIRO HASEBE ${ }^{2}$ and ATSUSHI OCHIAI ${ }^{1,2}$ \\ ${ }^{1}$ Laboratory of Cancer Biology, Department of Integrated Biosciences, Graduate School of Frontier Sciences, \\ The University of Tokyo, Kashiwa, Chiba; ${ }^{2}$ Pathology Division, Research Center for Innovative Oncology, \\ National Cancer Center Hospital East, 6-5-1 Kashiwanoha, Kashiwa, Chiba 277-8577, Japan
}

Received January 19, 2007; Accepted February 27, 2007

\begin{abstract}
Expression of human kallikrein 11 (hK11), secreted as a trypsin-like serine protease, is related to the prognosis of some human cancers, but its physiological functions in the steps of cancer progression are still unknown. In order to elucidate the enzymatic function of hK11 we investigated the substrate of hK11 and expression of KLK11, the gene encoding hK11. Among 20 human cancer cell lines tested, two estrogen receptor possessing $[\mathrm{ER}(+)]$ breast cancers showed the highest expression of KLK11. The investigation of the hK11 substrate showed that hK11 could degrade itself and insulin-like growth factor (IGF) binding protein 3 (IGFBP-3). Expression of KLK11 was detected in both human breast cancer tissue and in non-cancerous mammary glands, and significantly higher KLK11 expression was observed in histological grade I/II than in grade III breast cancers. The above results indicate that the hK11 expressed in ER(+) breast cancer cells may play a crucial role in breast cancer progression by increasing the bioavailability of IGFs via degradation of IGFBP-3.
\end{abstract}

\section{Introduction}

Tissue kallikreins are a group of secreted serine proteases, and are found in various species. In humans, the tissue kallikrein family consists of 15 homologous serine proteases encoded on chromosome 19q13-14. The kallikrein genes are referred to as $K L K 1-15$, and the proteins as hK1-15 (1). Human tissue kallikrein 11 (hK11), encoded by $K L K 11$, is a member of the family and was originally isolated as a trypsin-like serine protease (TLSP) by PCR of the human hippocampus (2).

Correspondence to: Dr Atsushi Ochiai, Pathology Division, Center for Innovative Oncology, National Cancer Center Hospital East, 6-5-1 Kashiwanoha, Kashiwa, Chiba 277-8577, Japan

E-mail: aochiai@east.ncc.go.jp

Key words: kallikrein 11, KLK11, hK11, IGFBP-3, breast cancer
Other family members including $K L K 11$ have three alternative splice variants (3-5). hK11 is expressed in a variety of normal tissues and fluids including prostate, stomach, trachea, skin, and colon tissues and milk, serum, and seminal plasma $(6,7)$. Recent studies revealed $K L K 11$ expression in prostate and breast cancer cell lines and in ovarian, prostate, breast, lung, pancreatic, colon, and neuroendocrine cancer tissue $(5,8,9)$. KLK11 expression has been found to be regulated by steroid hormones, especially estrogen $(6,10)$. Serum hK11 level has recently come to be regarded as a novel biomarker for ovarian cancer and prostate cancer $(8,10,11)$. In terms of its physiological function, hK11 has been found to cleave peptides after arginine $(3,4)$, but its physiological substrates are unknown.

Several epidemiological studies have reported that insulinlike growth factors (IGFs) are among the most important factors for cancer growth and cell survival in colon, prostate, and breast cancer (12). They are mainly expressed in a variety of tissues and affect the growth and survival of various cells for tissue maintenance. IGF binding proteins (IGFBPs) have high affinity for IGFs, and they modulate IGF function. Proteolysis of IGFBPs is an essential process by which cells increase IGF bioavailability. IGFBPs have been described as substrates of the MMP family, plasmin, thrombin, cathepsins, and some kallikreins, including hK2, hK3 (PSA), and hK4 $(1,13-16)$, but no studies have reported whether hK11 degrades IGFBPs and increases IGF bioavailability in human cancer cells.

Breast cancer is the most common cancer in women worldwide. Estrogen plays an important role not only in normal mammary gland development but in breast cancer progression, and estrogen has been a major therapeutic target in estrogen receptor (ER)-positive cancers (17). Breast cancer cells respond to IGF stimuli in addition to estrogen, and some studies have shown that IGF-I and IGF-II are potent mitogens for breast cancer cells (18). Several KLK family genes have been reported to be downregulated in breast cancer or related to the outcome of breast cancer, but no reports have ever described the relation between kallikrein 11 and breast cancer (19).

In the present study we examined the expression of KLK11 in various human cancer cell lines and investigated whether hK11 is capable of degrading IGFBP-3 and affecting IGF signals in human breast cancer cells. We also compared hK11 
expression with clinicopathological factors in human breast cancers.

\section{Materials and methods}

Proteins and reagents. Recombinant human kallikrein 11, IGFBP-3, and IGF-I were obtained from R\&D Systems (Minneapolis, MN).

Human breast cancer tissue. Tissue from 41 breast cancers and adjacent normal breast tissue from 32 of them were obtained from patients undergoing surgery for breast cancer in the National Cancer Center Hospital East Japan in 2002. The patients' median age was 55 years and the range was $27-83$ years. The histological grade was Grade I in 2 cases $(5 \%)$, Grade II in 22 cases (56\%), and Grade III in 16 cases (41\%). Twenty-four cases $(62 \%)$ were estrogen receptor (ER) positive and 21 cases $(54 \%)$ were progesterone receptor $(\mathrm{PgR})$ positive. There were 16 premenopausal patients (39\%). The specimens were homogenized in TRIzol reagent solution (Invitrogen, Carlsbad, CA) with a multi-bead shocker (Yasui kikai, Osaka, Japan) soon after surgical removal. A sample of each homogenate was stored at $-80^{\circ} \mathrm{C}$ until the RNA was extracted. All clinical and pathological information was extracted from the medical records.

Cell culture. Four human breast cancer cell lines, MCF-7, T47D, MDA-MB231, and MDA-MB468; eight human gastric cancer cell lines, MKN-28, MKN-45, HSC-43, HSC-44PE, HSC-57, HSC-58, HSC-59, and HSC-60; three prostate cancer cell lines, CWR22Rv1, PC3, and LNCaP; three human pancreatic cancer cell lines, Capan-1, MiaPaCa-2, and BxPC-3; a lung cancer cell line, A549; a colon cancer cell line, HT-29; and a human fibroblast cell line, MRC-5, were used in this study. The HSC-43, -44PE, -57, -58, -59, and -60 cells were gifts from Dr Yanagihara (Central Animal Laboratory, National Cancer Center Research Institute, Tokyo, Japan), and the others were obtained from the American Type Culture Collection (ATCC; Bethesda, MD). The MiaPaCa-2 and Capan-1 cells were grown in Dulbecco's modified Eagle's medium (DMEM, Sigma Aldrich, Taufkirchcen, Germany), and the others were grown in RPMI-1640 (Sigma Aldrich) containing 10\% heatinactivated fetal bovine serum (Equitech-Bio, Kerrville, TX). All cell lines were maintained under a humidified atmosphere containing $5 \% \mathrm{CO}_{2}$ at $37^{\circ} \mathrm{C}$. Subconfluent cells were sheared in $1 \mathrm{ml}$ of TRIzol reagent solution, and the homogenate was maintained at $-80^{\circ} \mathrm{C}$ until used.

RNA extraction and quantitative RT-PCR. Homogenized RNA samples were treated with $40 \mathrm{U}$ of RNase-free DNase I (Takara, Shiga, Japan) in $200 \mu 1$ of DEPC-treated water, 10X DNase buffer (Takara), and $40 \mathrm{U}$ of RNase Inhibitor (Toyobo, Osaka, Japan) at $37^{\circ} \mathrm{C}$ for $30 \mathrm{~min}$. All cDNAs were synthesized from $500 \mathrm{ng}$ of total RNA with an ExScript ${ }^{\mathrm{TM}}$ $\mathrm{RT}$ reagent kit (Takara) at $42^{\circ} \mathrm{C}$ for $15 \mathrm{~min}$ and then at $95^{\circ} \mathrm{C}$ for $2 \mathrm{~min}$. Quantitative real-time RT-PCR was carried out with a SmartCycler system (Cepheid, Sunnyvale, CA) and SYBR-green. In all, $1 \mu 1$ of cDNA solution corresponding to $50 \mathrm{ng}$ of total RNA was subjected to 45 PCR cycles of $5 \mathrm{sec}$ at $95^{\circ} \mathrm{C}, 20 \mathrm{sec}$ at $60^{\circ} \mathrm{C}$, and $6 \mathrm{sec}$ at $83^{\circ} \mathrm{C}$ in a $25-\mu 1$ mixture containing $12.5 \mu 12 X$ SYBR Premix Ex Taq (Takara) and $0.2 \mu \mathrm{M}$ each of forward and reverse gene-specific primers. Expression of the housekeeping gene (GAPDH: glyceraldehyde-3-phosphate dehydrogenase) was measured under the same experimental conditions. The KLK11 forward primer was 5'-GCAATGACATCATGCTGGTGAAG-3', and the reverse primer was 5'-ATGTTGGCGCATCGCAAG-3'. The GAPDH forward primer was 5'-GCACCGTCAAGGCTGAG AAC-3', and the reverse primer was 5'-ATGGTGGTGAAG ACGCCAGT-3'. The KLK11 primers are specific for isoform 1. The amplification specificity of the PCR products was confirmed by melting curve analysis and $2 \%$ agarose gel electrophoresis. An external standard curve for each gene was generated by using 102-fold dilutions of PCR products corresponding to $1 \times 10^{8}-1 \times 10^{2}$ copies.

Autocleavage assays. Recombinant pro-hK11 was activated by incubation with thermolysin for 15 or $30 \mathrm{~min}$ at $37^{\circ} \mathrm{C}$ in a preincubation buffer $\left[50 \mathrm{mM}\right.$ Tris $(\mathrm{pH} 6.8), 10 \mathrm{mM} \mathrm{CaCl}_{2}$, and $1 \mathrm{M} \mathrm{NaCl}$. The ratio of thermolysin to hK11 was 1:40. After the preincubation, the thermolysin activity was inhibited by adding $10 \mathrm{mM}$ EDTA, and active hK11 was incubated for

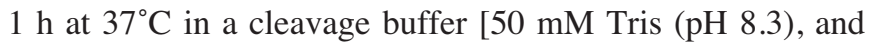
$10 \mathrm{mM}$ EDTA]. A 20-mM phenylmethylsulfonyl fluoride (PMSF) solution was used as an hK11 inhibitor. 2-Mercaptoethanol was added to provide reducing conditons, and the reaction solution was boiled and then resolved by SDS-PAGE.

Western blot analysis. hK11 aliquots were subjected to SDSPAGE on $12 \%$ polyacrylamide gels under reducing or nonreducing conditions, and then transferred to a polyvinylidene difluoride (PVDF) membrane (Millipore, Bedford, MA). Non-specific binding was blocked by exposure to $5 \%$ nonfat milk powder and 1\% BSA in PBS (pH7.4) containing $0.1 \%$ Tween-20 for $1 \mathrm{~h}$ at room temperature. The membrane was incubated overnight in mouse monoclonal anti-human kallikrein 11 antibody (1:500; clone 189308; R\&D Systems) at $4^{\circ} \mathrm{C}$, and then for $1 \mathrm{~h}$ with peroxidase-labeled rabbit antimouse antibody (1:3000; Zymed Laboratories, San Francisco, CA). The hK11 bands were visualized with ECL chemiluminescent reagent (Amersham Corp., Arlington Heights, IL).

IGFBP-3 cleavage assays. Recombinant human IGFBP-3 was incubated with active hK11 that had been preincubated with thermolysin for $15 \mathrm{~min}$ as described above, in a cleavage buffer [50 mM Tris (pH 7.5) and $10 \mathrm{mM}$ EDTA] for 4-24 h at $37^{\circ} \mathrm{C}$ (Substrate enzyme ratio $=20: 1$ ). IGF-I was tested for inhibition of proteolytic activity by incubation with IGFBP-3 and active hK11. The molar ratio of IGF-I to IGFBP-3 was 1:1. A $20 \mathrm{mM}$ PMSF solution was used as an hK11 inhibitor, and the reactions were terminated by the addition of sample buffer containing the reducing agent 2-mercaptoethanol. The reaction mixture was then separated on $15 \%$ SDS-PAGE and transferred onto PVDF. IGFBP-3 fragments were analyzed by Western blot analysis as described above by using a goat polyclonal anti-human IGFBP-3 antibody (1:500; C-19; Santa Cruz Biotechnology, Santa Cruz, CA) and peroxidaselabeled rabbit anti-goat antibody (1:6000; Zymed Laboratories), and they were visualized with Lumi-Lightplus Western blotting substrate (Roche, Mannheim, Germany). 


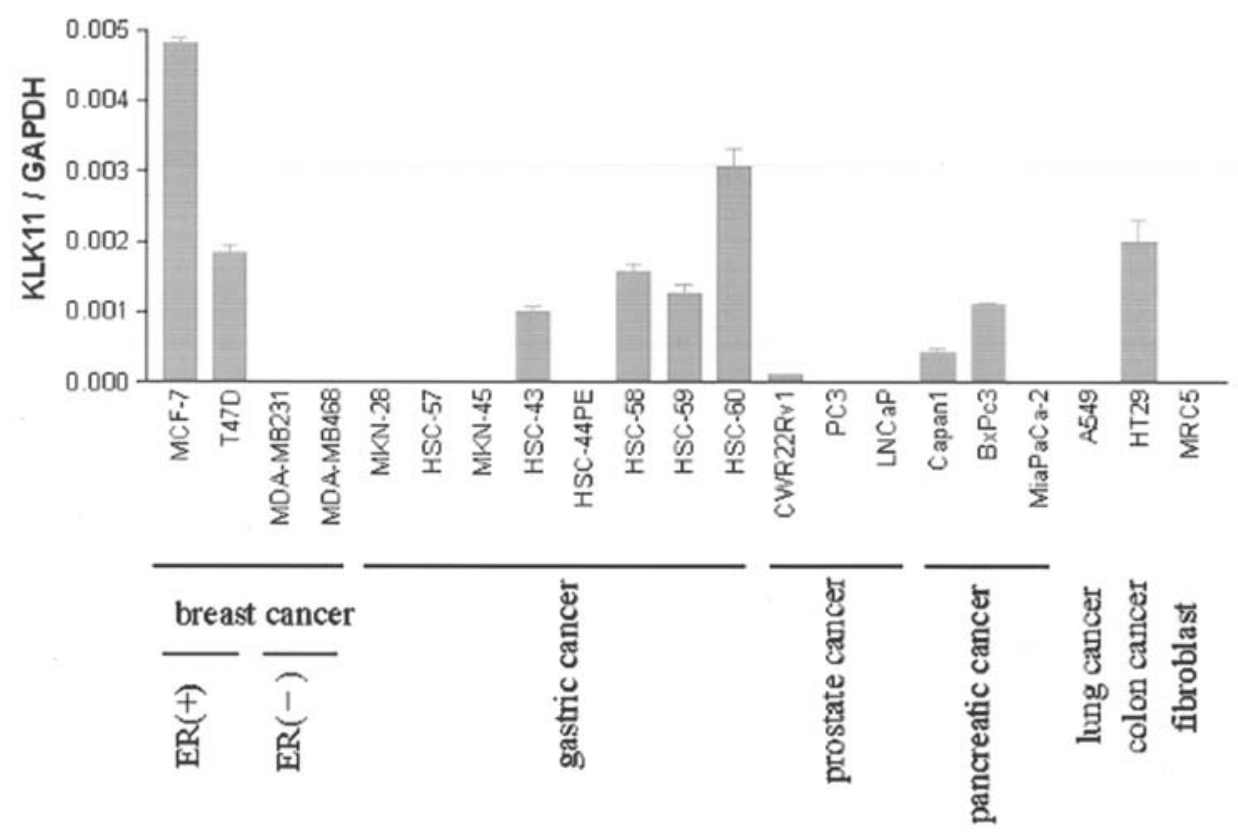

Figure 1. Quantification of KLK11 gene expression in 21 human cell lines (20 cancer and 1 normal) by real-time RT-PCR. All KLK11 expression amounts were corrected by the expression amounts of housekeeping gene, GAPDH.

Statistical analysis. The paired t-test was used for comparison between cancerous lesions and non-cancerous lesions. Since KLK11 mRNA expression was asymmetrically distributed, the relationship between expression of KLK11 and cancer histology was assessed by linear regression analysis (MannWhitney U test). Significance was defined as $\mathrm{p}<0.05$.

\section{Results}

Expression of KLK11 in ER(+) breast cancer cell lines. The results for expression of the KLK11 gene in 20 human cancer cell lines and 1 human normal cell line obtained by real-time RT-PCR are shown in Fig. 1. Various levels of KLK 11 expression were observed in the breast (2/4), gastric (4/8), colon $(1 / 1)$, pancreatic $(2 / 3)$, and prostate $(1 / 3)$ cancer cell lines. No expression was detected in the lung cancer cell line or the normal fibroblastic cell line. KLK11 was expressed in 2 breast cancer cell lines, MCF-7 and T47D, both of which are estrogendependent, but not in either of the 2 estrogen-independent cell lines, MDA-MB231 and MDA-MB468. KLK11 was expressed in all 4 gastric cancer cell lines, HSC-43, HSC-58, HSC-59, and HSC-60, all of which derived from scirrhous gastric cancers. Colon cancer cell line HT-29, pancreatic cancer cell lines BxPC3 and Capan1, and prostate cancer cell line CWR22Rv1 also expressed $K L K 11$, but the levels of expression were lower than in the estrogen-dependent breast cancer cell lines.

Search for hK11 substrates and hK11 auto-degradation. First, thermolysin-activated hK11 was tested for ability to catalyze the degradation of synthetic fluorogenic peptides. The results confirmed its enzymatic ability and the results of previous studies showing that hK11 has activity that cleaves peptides after arginine (data not shown) (3). When the active hK11 alone was incubated in cleavage buffer, a $20-\mathrm{kDa}$ band was detected by Western blotting in addition to the main $40-\mathrm{kDa}$ band, but it was not detected under non-reducing conditions with SDS-
PAGE (Fig. 2A). In addition, under reducing conditions only thermolysin-activated hK11 cleaved itself, and hK11 did not display auto-activating ability (lanes 3 and 7). The proteolysis reaction was preincubation time-dependent (lanes 1 and 3), and the reaction was inhibited by the serine protease inhibitor PMSF (lanes 1 and 5, and lanes 1 and 2) (Fig. 2B). These findings indicate that thermolysin-activated $\mathrm{hK} 11$ is not naturally degraded but actively degrades itself, and that the two peptides that are cleaved are held together by internal disulfide bonds.

Detection of IGFBP-3 degradation by $h K 11$. It is well known that IGF signals play a crucial role in the progression of breast cancer and that the IGF activity is regulated by degradation of IGFBPs by IGFBP proteases, including other hK family members, such as PSA (hK3). The hK11 enzymatic activity against IGFBP was examined in vitro, and Western blot analysis after incubation for $4 \mathrm{~h}$ revealed that IGFBP-3 was cleaved into $325-\mathrm{kDa}, 20-\mathrm{kDa}$, and $17-\mathrm{kDa}$ fragments by hK11. The hK11 induced IGFBP-3 degradation was inhibited by PMSF, indicating that the serine protease activity of hK11 is responsible for IGFBP-3 cleavage. These results demonstrate that hK11 functions as an IGFBP-3 protease (Fig. 2C). The degradation of IGFBP-3 by hK11 was unaffected by coupling of IGFBP-3 with IGF-I (Fig. 2F).

A time-course analysis was performed to further characterize the cleavage of IGFBP-3 by hK11. hK11 cleaved IGFBP-3 into three fragments in a time-dependent manner. All three fragments were observed after incubation for $6 \mathrm{~h}$ but only the smallest fragment was detected after incubation for more than $6 \mathrm{~h}$. By $24 \mathrm{~h}$, almost all of the intact IGFBP-3 had been degraded (Fig. 2E). IGFBP-3 did not self-degrade, and was not degraded by treatment with thermolysin in EDTAcontaining buffer, even for $24 \mathrm{~h}$ (Fig. 2D).

KLK11 expression in normal human tissue and human cancer tissue, and relation to clinicopathological variables. KLK11 


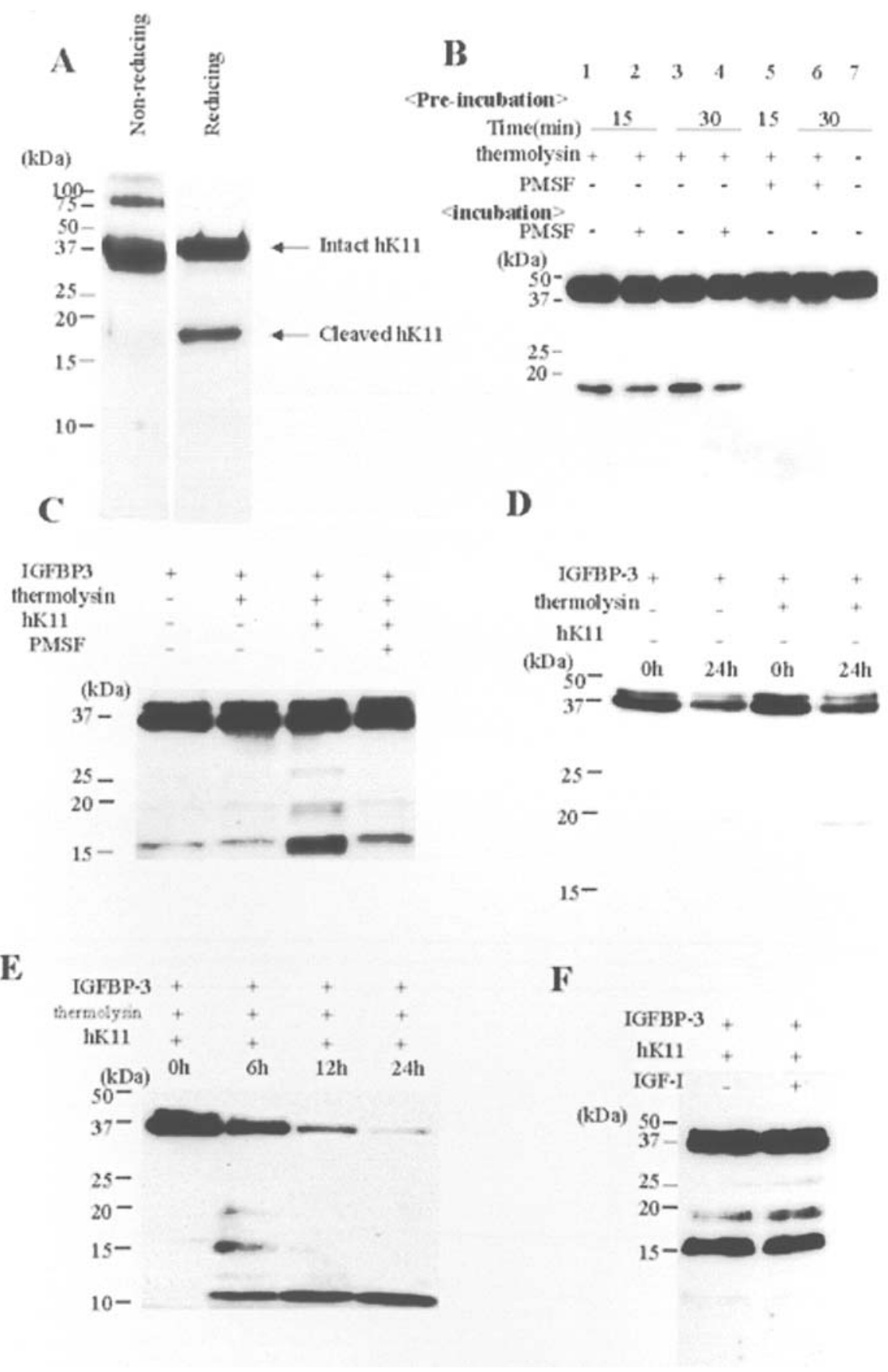

Figure 2. hK11 degrades itself and IGFBP-3. (A) Incubated rhK11 was separated by SDS-PAGE under non-reducing or reducing conditions, and detected by anti-kallikrein $11 \mathrm{mAb}$. Intact and cleaved hK11 are indicated by arrows. (B) Thermolysin-activated or non-activated rhK11 was incubated with or without serine protease inhibitor PMSF. Then SDS-PAGE was performed under reducing conditions and detected by anti-kallikrein $11 \mathrm{mAb}$. (C) Western blot analysis by IGFBP-3 Ab. The time-course of IGFBP-3 degradation without hK11 (D) and by hK11 (E). (F) Effect of added IGF-I on hK11 proteolysis of IGFBP-3. The molar ratio of IGF-I to IGFBP-3 was 1:1.

mRNA expression was analyzed in cancer tissue from 41 breast cancers and from adjacent normal breast tissue in 32 of the surgical specimens. KLK11 expression was detected in both the cancer tissue and normal tissue, and there was no significant difference between them. The KLK11 expression level in the breast cancer tissue of 42 cancer patients was compared to the other clinicopathological factors, such as estrogen and progesterone receptor status, histological grade, and menopausal status. Significantly higher $K L K 11$ expression was found in the Grade I/II patients $(\mathrm{p}<0.05)$. KLK11 tended to be expressed in patients with estrogen-rich cancers, such as in $\mathrm{ER}(+)$ cancers, $\operatorname{PgR}(+)$ cancers, and cancers from premenopausal patients, but no significant association was found between the relatively estrogen-rich condition and KLK11 expression (Fig. 3).

\section{Discussion}

Several studies have pointed to KLK11 expression or the serum hK11 level as a potential biomarker for prostate cancer and ovarian cancer, the same as other kallikrein family proteins $(1,8,10,11)$. Since all $K L K$ genes, including $K L K 11$, are 
A

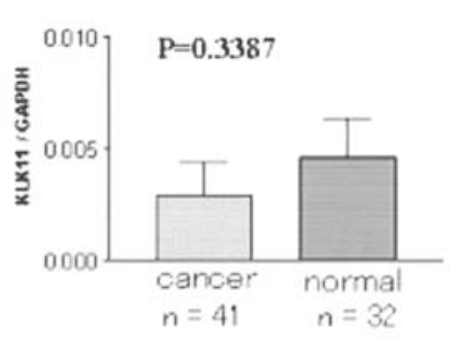

D

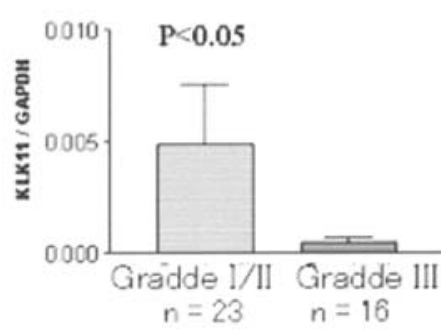

B

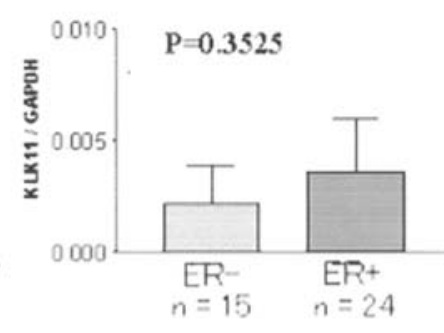

C

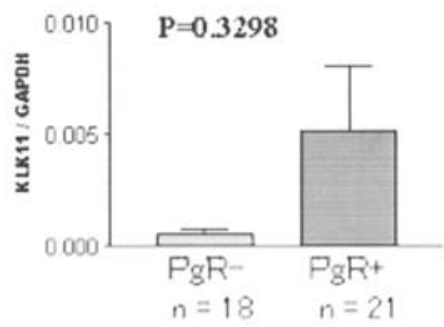

E

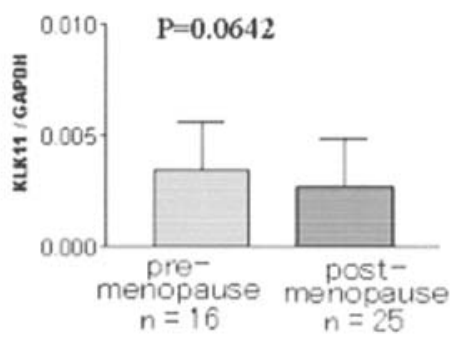

Figure 3. Expression of $K L K 11$ in human breast tissues and its relation to clinicopathological variables. (A) $K L K 11$ expression in RNA samples from 41 breast cancer tissues and 32 normal breast tissues was quantified by real-time RT-PCR. Then, the expression of KLK11 in cancer lesions was compared with established prognostic factors: (B), ER; (C), PgR; (D), histological type; and (E), menopausal.

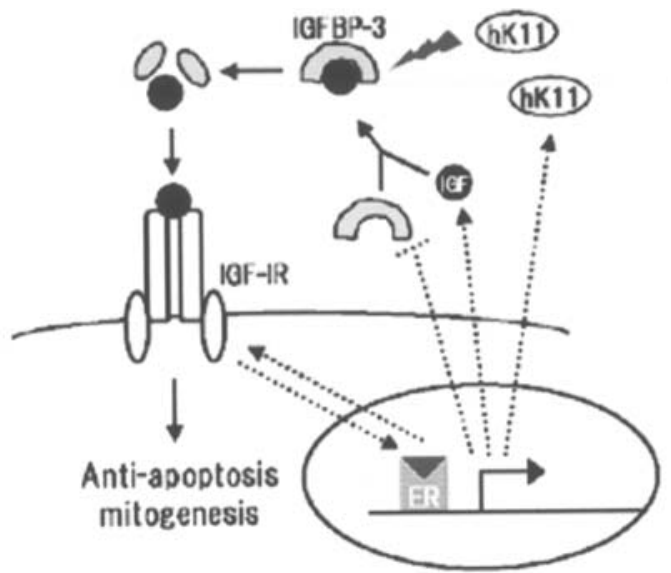

Figure 4. Model of IGF-estrogen-hK11 crosstalk in breast cancer cell. The dotted lines indicate the crosstalk referred to in previous reports.

under steroid hormone regulation (1), the hormone-regulated kallikrein family may be involved in sex-hormone-related tissues and cancers, such as breast, ovary, prostate, and so on. We investigated KLK11 expression in 21 human cell lines and found that $K L K 11$ was highly expressed in $2 \mathrm{ER}(+)$ breast cancer cell lines. Most members of the kallikrein family, but not hK11, have been reported to be involved in breast cancer (19). KLK11 expression was observed in breast cancer tissue as well as in normal breast tissue. Significantly higher KLK11 expression in breast cancer tissue was found in low histological grade breast cancers and $K L K 11$ expression seemed to be lost as the degree of malignancy increases. Estrogen-rich cancer tissue, such as $\mathrm{ER}(+)$ cancer tissue, $\operatorname{PgR}(+)$, cancer tissue, and cancer tissue from premenopausal patients, tends to express $K L K 11$, although no significant correlation was found.
It is known that low histological grade cancer tissue often expresses estrogen and progesterone receptors (20). These results indicate that estrogen-regulated KLK11 expression can be used as a differentiation marker for early-stage breast cancer. This is the first report on the relationship between breast cancer and $K L K 11$. hK11 may also be related to the development of breast cancer through IGF activation. Since a large amount of IGF is stored in the bone microenvironment (21), KLK11 may contribute to the bone metastasis that occurs beginning with early-stage breast cancer. By contrast, relatively high $K L K 11$ expression was found in human cell lines derived from scirrhous gastric cancer. There have been some reports that scirrhous gastric cancer tends to occur in postpregnancy young women (22). The relationship between estrogen and scirrhous gastric cancer has been discussed for many years, but no consensus has been reached. The higher KLK11 expression in the scirrhous gastric cancer cell lines indicates that KLK11 may be involved in the development of scirrhous gastric cancer and its distinctive fibrosis via estrogen.

Although KLK11 was discovered in 1998 (2), the substrates and enzymatic activity of hK11 have never been demonstrated. hK11 has been reported not to cleave hK3, hK5, plasminogen, kininogen, or collagen type I to IV, which are degraded by some other kallikreins (23). Thus, we searched hK11 substrates by a cleavage assay, and demonstrated that hK11 actively degrades itself. The cleavage yields two peptides of approximately $20 \mathrm{kDa}$ that are held together by internal disulfide bonds. This $20-\mathrm{kDa}$ autocleavage product is the same as the previously reported 20-kDa inactive hK11 in seminal plasma. That report stated that plasmin appeared to cleave hK11 (23), but in the present study we observed auto-cleavage activity that was stronger than plasmin cleavage. Thus, this autodegradation seems to occur in vivo and to regulate hK11 enzymatic activity through inactivation by internal cleavage, 
which is a common mechanism in the human kallikrein family. $\mathrm{hK} 2, \mathrm{hK} 4, \mathrm{hK} 6$, and hK13 are capable of self-proteolysis and self-activation, and some family members auto-degrade and inactivate themselves (1). Although no auto-activation was observed in this study, the auto-degradation ability observed was similar. Since no inhibitor of hK11 has been found, hK11 may regulate its own enzymatic activity.

The present study clearly demonstrated that hK11 cleaves IGFBP-3. IGF is a major growth factor that affects cell survival and apoptosis, and its bioavailability depends on the affinity of IGFBPs for IGF. IGFBP-3 is mainly produced by liver mesenchymal cells and is the most abundant IGFBP in serum (13). In human breast cancer IGF-I is secreted by the stroma, and IGF-II is secreted by the stroma and malignant breast cancer cells. Some reports have shown that crosstalk occurs between estrogen and the IGF system in human breast cancer. Estrogen upregulates transcription of IGF-I, IGF-II, and IGF-I receptor, but downregulates expression of IGFBPs. IGF-I increases expression of the ER-responsive gene and enhances the transcriptional activity of ER (18), and IGFBP-3 protease $\mathrm{hK} 11$ is expressed under hormone control. These findings suggest that estrogen, the IGF system, and hK11 may interact with each other and promote cancer cell survival in breast cancer (Fig. 4). The IGF-steroid hormone-kallikrein system may also function in hormone-regulated cancers, such as prostate cancer and ovarian cancer.

In conclusion, our study has demonstrated that hormoneregulated hK11 functions as an IGFBP-3 protease and may contribute to the modulation of IGF bioavailability in the breast cancer microenvironment.

\section{Acknowledgments}

We thank Dr S. Yoshida (Department of Functional Anatomy and Neuroscience, Asahikawa Medical College, Hokkaido, Japan) for his kind advice, and C. Okumura, Y. Okuhara, M. Okumoto, and H. Hashimoto for their technical assistance. The present study was supported in part by Grant-in-Aid for Cancer Research from the Ministry of Health, Labor and Welfare (16-15) and by Grant-in-Aid for the third-term Comprehensive 10-year strategy for Cancer Control from the Ministry of Health, Labor and Welfare in Japan.

\section{References}

1. Borgono CA, Michael IP and Diamandis EP: Human tissue kallikreins: physiologic roles and applications in cancer. Mol Cancer Res 2: 257-280, 2004.

2. Yoshida S, Taniguchi M, Suemoto T, Oka T, He X and Shiosaka S: cDNA cloning and expression of a novel serine protease, TLSP. Biochim Biophys Acta 1399: 225-228, 1998.

3. Mitsui S, Yamada T, Okui A, Kominami K, Uemura H and Yamaguchi N: A novel isoform of a kallikrein-like protease, TLSP/hippostasin, (PRSS20), is expressed in the human brain and prostate. Biochem Biophys Res Commun 272: 205-211, 2000.
4. Nakamura T, Mitsui S, Okui A, Miki T and Yamaguchi N: Molecular cloning and expression of a variant form of hippostasin/ KLK11 in prostate. Prostate 54: 299-305, 2003.

5. Nakamura T, Mitsui S, Okui A, et al: Alternative splicing isoforms of hippostasin (PRSS20/KLK11) in prostate cancer cell lines. Prostate 49: 72-78, 2001.

6. Yousef GM, Chang A, Scorilas A and Diamandis EP: Genomic organization of the human kallikrein gene family on chromosome 19q13.3-q13.4. Biochem Biophys Res Commun 276: 125-133, 2000.

7. Komatsu N, Saijoh K, Toyama T, et al: Multiple tissue kallikrein mRNA and protein expression in normal skin and skin diseases. Br J Dermatol 153: 274-281, 2005.

8. Shigemasa K, Gu L, Tanimoto H, O'Brien TJ and Ohama K: Human kallikrein gene 11 (KLK11) mRNA overexpression is associated with poor prognosis in patients with epithelial ovarian cancer. Clin Cancer Res 10: 2766-2770, 2004.

9. Bhattacharjee A, Richards WG, Staunton J, et al: Classification of human lung carcinomas by mRNA expression profiling reveals distinct adenocarcinoma subclasses. Proc Natl Acad Sci USA 98: 13790-13795, 2001.

10. Diamandis EP, Okui A, Mitsui S, et al: Human kallikrein 11: a new biomarker of prostate and ovarian carcinoma. Cancer Res 62: 295-300, 2002.

11. Stavropoulou P, Gregorakis AK, Plebani M and Scorilas A: Expression analysis and prognostic significance of human kallikrein 11 in prostate cancer. Clin Chim Acta 357: 190-195, 2005.

12. Le Roith D and Roberts CT Jr: The insulin-like growth factor system and cancer. Cancer Lett 195: 127-137, 2003.

13. Firth SM and Baxter RC: Cellular actions of the insulin-like growth factor binding proteins. Endocr Rev 23: 824-854, 2002.

14. Miyamoto S, Yano K, Sugimoto S, et al: Matrix metalloproteinase-7 facilitates insulin-like growth factor bioavailability through its proteinase activity on insulin-like growth factor binding protein 3. Cancer Res 64: 665-671, 2004

15. Nakamura M, Miyamoto S, Maeda $\mathrm{H}$, et al: Matrix metalloproteinase-7 degrades all insulin-like growth factor binding proteins and facilitates insulin-like growth factor bioavailability. Biochem Biophys Res Commun 333: 1011-1016, 2005.

16. Matsumura M, Bhatt AS, Andress D, Clegg N, Takayama TK, Craik CS and Nelson PS: Substrates of the prostate-specific serine protease prostase/KLK4 defined by positional-scanning peptide libraries. Prostate 62: 1-13, 2005.

17. Osborne CK and Schiff R: Estrogen-receptor biology: continuing progress and therapeutic implications. J Clin Oncol 23: 1616-1622, 2005

18. Sachdev D and Yee D: The IGF system and breast cancer. Endocr Relat Cancer 8: 197-209, 2001

19. Yousef GM, Yacoub GM, Polymeris ME, Popalis C, Soosaipillai A and Diamandis EP: Kallikrein gene downregulation in breast cancer. Br J Cancer 90: 167-172, 2004.

20. Thoresen S, Thorsen T, Tangen M and Hartveit F: Oestrogen and progesterone receptor content and the distribution of histological grade in breast cancer. Breast Cancer Res Treat 2: 251-255, 1982.

21. Govoni KE, Baylink DJ and Mohan S: The multi-functional role of insulin-like growth factor binding proteins in bone. Pediatr Nephrol 20: 261-268, 2005.

22. Jaspers VK, Gillessen A and Quakernack K: Gastric cancer in pregnancy: do pregnancy, age or female sex alter the prognosis? Case reports and review. Eur J Obstet Gynecol Reprod Biol 87: 13-22, 1999.

23. Luo LY, Shan SJ, Elliott MB, Soosaipillai A and Diamandis EP: Purification and characterization of human kallikrein 11, a candidate prostate and ovarian cancer biomarker, from seminal plasma. Clin Cancer Res 12: 742-750, 2006. 